\title{
Vorwort und Überblick zum Sonderheft PISA Plus 2012-2013
}

\author{
Jörg-Henrik Heine
}

Online publiziert: 9. Mai 2017

(C) Springer Fachmedien Wiesbaden 2017

Das vorliegende Sonderheft präsentiert in insgesamt elf Beiträgen die Befunde zu der Ergänzungsstudie PISA Plus 2012-2013. Es handelt sich um eine zusätzliche Erhebung in Deutschland im Jahr 2013 als Ergänzung der fünften internationalen Vergleichsstudie Programme for International Student Assessment (PISA) der OECD. PISA Plus 2012-2013 ist damit eine längsschnittliche Ergänzung zu PISA 2012 (vgl. Prenzel et al. 2013), mit welcher die Kompetenzentwicklung der Schülerinnen und Schüler in Deutschland im Verlauf eines Schuljahres erfasst werden soll. Ausgangspunkt war die klassenbasierte Stichprobe im Rahmen der internationalen Erhebung im Jahr 2012, die $n=9998$ Schülerinnen und Schüler aus 222 Schulen umfasste. Dieselben Schülerinnen und Schüler, welche im Rahmen der internationalen Erhebung teilgenommen hatten, wurden ein Jahr später im Jahr 2013 in der zehnten Jahrgangsstufe erneut getestet.

Ein häufiger Kritikpunkt an den international vergleichenden PISA Studien ist deren rein querschnittliches Forschungsdesign, welches aus wissenschaftstheoretischer Perspektive keine kausalen Aussagen über Prädiktoren für die Kompetenzentwicklung der Schülerinnen und Schüler zulässt (z. B. Diekmann 2014; Kromrey 2002). Lediglich in einem sehr eingeschränkten Umfang lassen sich auf Basis der internationalen Daten, im Rahmen der Trendanalysen über mehrere Zyklen hinweg, Aussagen zu Entwicklungstendenzen der Leistungsfähigkeit eines Bildungssystems als Ganzes treffen (vgl. dazu auch Heine et al. 2016). Eine erweiterte Analyse und Erkenntnisse zu differenzierten Bedingungsgefügen als Antezedens schulischer Kompetenzentwicklung sind oft nicht möglich oder müssen zumindest unsicher bleiben. Diese empirische Lücke soll die deutsche Ergänzungsstudie PISA Plus 2012-2013

\footnotetext{
J.-H. Heine $(\bowtie)$

Technische Universität München School of Education, München, Zentrum für internationale Bildungsvergleichsstudien (ZIB), Arcisstraße 21, 80333 München, Deutschland

E-Mail: joerg.heine@tum.de
} 
schließen. Am Standort Technische Universität München (TUM) des Zentrums für internationale Bildungsvergleichsstudien (ZIB), das seit 2010 mit der Koordination der PISA-Studien in Deutschland befasst ist, wurde daher eine zweite Erhebung für die Kohorte PISA 2012 vorbereitet. Das primäre Ziel bestand in der Erfassung von Kompetenzveränderungen von Schülerinnen und Schülern innerhalb eines Schuljahres in den drei PISA-Domänen Mathematik, Naturwissenschaften und Lesen. Neben der längsschnittlichen Erweiterung der internationalen PISA-Hauptstudie integriert das Projekt PISA Plus 2012-2013 auch zusätzlich erhobene Daten wie beispielsweise kognitive Basisfertigkeiten im Bereich Leseverständnis. Diese Ergänzungsstudien wurden am ZIB-Standort Frankfurt beim Deutschen Institut für Internationale Pädagogische Forschung (DIPF) vorbereitet und begleitet.

Zur Erfassung der drei Kompetenzbereiche wurden Aufgaben aus den internationalen PISA-Tests, aber auch Aufgaben aus dem Kompetenz-Test zur Überprüfung der Bildungsstandards aus dem Ländervergleich des Instituts zur Qualitätsentwicklung im Bildungswesen (IQB) verwendet (vgl. Pant et al. 2013). Die vorliegende Datengrundlage bietet daher die Möglichkeit einer vergleichenden Darstellung von Entwicklungsverläufen anhand der jeweiligen Operationalisierungen für schulische Kompetenzen. Während die PISA-Aufgaben die schulischen Kompetenzen eher curricular übergreifend erfassen, bilden die Aufgaben aus dem IQB-Ländervergleich die Bildungsstandards für Deutschland eher curricular spezifisch ab. Eine vergleichende Darstellung der Kompetenzverläufe für den Bereich Mathematik in Abhängigkeit der beiden Operationalisierungen, wird beispielsweise im Beitrag 1. Veränderung der mathematischen Kompetenz von der neunten zur zehnten Klassenstufe (Lehner et al. 2017), referiert. Damit verbunden, bieten die Daten aus PISA Plus 2012-2013 die Möglichkeit der Frage nachzugehen, ob Zusammenhänge zwischen den Kompetenzwerten aus den beiden Operationalisierungen bestehen. Darauf aufbauend kann überprüft werden, ob sich die beiden Messwerte gegebenenfalls über ein „Linking“ verknüpfen lassen. Dieser Frage wird in Beitrag 2. Äquivalenz der Erfassung mathematischer Kompetenzen in PISA 2012 und im IQB-Ländervergleich 2012 (Ehmke et al. 2017a) nachgegangen - ebenfalls anhand der Schwerpunktdomäne mathematische Kompetenz.

Wie bereits in der längsschnittlichen PISA-Ergänzung für die Jahre 2003-2004 (vgl. PISA-Konsortium Deutschland 2006), ermöglicht die Erweiterung des internationalen PISA-Untersuchungsdesigns um einen zweiten Messzeitpunkt die Suche nach kausal bedeutsamen Einflussgrößen und Bedingungsfaktoren der Kompetenzveränderung. Im Dezember 2012 wurden sämtliche Schulen, welche bereits an der internationalen Erhebung teilgenommen hatten, erneut eingeladen, freiwillig mit den gleichen Klassen sowie Schülerinnen und Schülern an einer weiteren Erhebung im Frühjahr 2013 teilzunehmen. Von der Ausgangsstichprobe im Jahr 2012 (222 Schulen) erklärten sich insgesamt 148 Schulen bereit, an einer zweiten Erhebung mitzuwirken. Insgesamt liegen von diesen Schulen für $n=4871$ Schülerinnen und Schüler Daten zu der Messwiederholung vor. Die grundsätzliche Freiwilligkeit einer wiederholten Teilnahme auf der Ebene der Schulen als auch auf der Ebene der Schülerinnen und Schüler stellt aus der Perspektive der Stichprobenplanung und im Hinblick auf die Verallgemeinerbarkeit der darauf basierenden Befunde eine methodische Herausforderung dar. So mussten zur Wahrung der Repräsentativität der 
Daten neue Fall- und Replikat-Gewichte für die neu zusammengesetzte Stichprobe bei der IEA Hamburg, seit Beginn der PISA-Studie Subkontraktor der nationalen Studienleitung, errechnet werden. Die Zusammensetzung der Stichprobe zu den beiden Messzeitpunkten und der notwendigerweise zu analysierende Mechanismus des Stichprobenausfalls wird in Beitrag 11. Empirische Grundlage, Stichprobenausfall und Adjustierung im PISA-Längsschnitt 2012-2013 (Heine et al. 2017) des vorliegenden Heftes berichtet. Im Zentrum der Betrachtung des Projektes stand die Darstellung von Entwicklungsverläufen innerhalb des Schuljahres 2012/2013 im Kompetenzbereich Mathematik.

Im Laufe der Jahre 2014 und 2015 wurden die erhobenen Daten am ZIB-Standort Kiel durch das Leibniz-Institut für die Pädagogik der Naturwissenschaften und Mathematik (IPN) auf einer gemeinsamen Metrik skaliert. Aufgrund der komplexen Datenlage musste dabei teilweise ,methodisches Neuland“ betreten werden. Die Komplexität der Daten resultierte einerseits aus der Integration zweier Operationalisierungen für schulische Kompetenzen in einem neu zusammengestellten TestheftDesign und andererseits aus dem empirischen Befund eines systematischen Verlustes des Stichprobenumfangs zum zweiten Messzeitpunkt. Die Methodik der Skalierung der PISA Kompetenzmesswerte wird im vorliegenden Heft in Beitrag 9. IRT-Skalierung der PISA-Tests im PISA-Längsschnitt 2012/2013: Auswirkungen von Testkontexteffekten auf die Zuwachsschätzung (Nagy et al. 2017b) dargestellt. In Ergänzung dazu werden die methodischen Aspekte der Skalierung der Bildungsstandards im Beitrag 10. Längsschnittskalierung der Tests zur Überprüfung des Erreichens der Bildungsstandards der Sekundarstufe I im PISA-Längsschnitt 2012/2013 (Nagy et al. 2017a) referiert.

In Erweiterung der längsschnittlichen Untersuchung in den Jahren 2003-2004 wurde bei PISA Plus 2012-2013 zusätzlich die Erfassung der Kompetenzentwicklung in den beiden Nebendomänen Naturwissenschaften und Lesen berücksichtigt. Für den Bereich Naturwissenschaften werden die Befunde aus entsprechenden Analysen im Beitrag 6. Die Veränderung der naturwissenschaftlichen Kompetenz von der 9. zur 10. Klasse bei PISA und dem Ländervergleich unter Berücksichtigung von geschlechts- und schulartspezifischen Unterschieden sowie der Zusammensetzung der Schülerschaft (Schiepe-Tiska et al. 2017) berichtet. Die Entwicklung der Lesekompetenz wird unter Berücksichtigung von Positionseffekten der einzelnen Lese-Aufgaben im Beitrag 7. Veränderungen der Lesekompetenz von der 9. zur 10. Klasse: Differenzielle Entwicklungen in Abhängigkeit der Schulform und des soziodemografischen Hintergrunds? (Nagy et al. 2017c) dargestellt. Die Beiträge beziehen dabei zentrale Kontextvariablen wie beispielsweise Geschlecht und Schulart in Ihre Analysen zur Kompetenzentwicklung ein. Solche Klima- und Kontextvariablen können im Rahmen einer längsschnittlichen Betrachtung die Grundlage für Erklärungsmodelle der gemessenen Kompetenzveränderung darstellen. Die bei PISA Plus 2012-2013 erhobenen Variablen beziehen sich hierbei auf die Ebene der Schulen, der Schulklassen sowie der einzelnen Schülerinnen und Schüler. Dies erlaubt die Analyse von Bedingungen, die den Kompetenzerwerb beeinflussen und kann Erkenntnisse über relevante Einflussfaktoren der Kompetenzentwicklung von Schülerinnen und Schülern generieren. PISA Plus 2012-2013 bietet damit zum Beispiel die Möglichkeit, Aussagen über die Wirksamkeit des Unterrichts am Ende der Sekundarstufe I zu 
überprüfen. Der Beitrag 3. Mathematikunterricht und Schülerleistung in der Sekundarstufe: Zur Validität von Schülerbefragungen in Schulleistungsstudien (Kuger et al. 2017) untersucht im Hinblick auf die Kompetenzentwicklung im Bereich Mathematik, die Bedeutung von verschiedenen Unterrichtsmerkmalen, Unterrichtsaktivitäten, der Unterrichtsqualität sowie der Unterrichtsinhalte.

Ein im Zusammenhang mit internationales Schulleistungsstudium immer wieder diskutierter Aspekt bezieht sich auf die Auswirkungen sozialer und zuwanderungsbezogener Unterschiede auf schulische Kompetenzen. Dieses Thema wird im Hinblick auf die Kompetenzentwicklung für den Bereich der mathematischen Kompetenz im Beitrag 5. Einfluss sozialer und zuwanderungsbezogener Disparitäten sowie der Klassenkomposition auf die Veränderung der mathematischen Kompetenz von der neunten zur zehnten Klassenstufe (Kiemer et al. 2017) aufgegriffen und diskutiert. Eine Sonderstellung in Bezug auf die zugrundeliegende Datenbasis nimmt Beitrag 8. Der Einfluss kognitiver Basisfertigkeiten auf die Änderung der in PISA gemessenen Lesekompetenz (Hahnel et al. 2017) ein. Hier beziehen sich die Analysen nicht auf den klassenbasierten Datensatz, sondern auf die Teilstichprobe der 15-jährigen Schülerinnen und Schüler. Der Beitrag widmet sich auf der Ebene der Schülerinnen und Schüler der Analyse von Zusammenhängen zwischen basalen Prozessen des Leseverstehens und der Lesekompetenz gemäß der Definition in der theoretischen Rahmenkonzeption sowie deren Veränderung im Verlauf eines Schuljahres.

Ein insbesondere im schulischen Bildungssystem in Deutschland immer wieder auch kontrovers diskutierter Aspekt bezieht sich auf die Sinnhaftigkeit und die Auswirkungen von Klassenwiederholungen. In den vorliegenden Daten aus dem Projekt PISA Plus 2012-2013 können durch die wiederholte Erhebung derselben Stichprobe - wenn auch nur in vergleichsweise geringem Umfang $(n=89)$ - Schülerinnen und Schüler identifiziert werden, die im Schuljahr 2012/2013 die entsprechende Klassenstufe wiederholt haben. Auf dieser empirischen Basis untersucht der Beitrag 4. Kompetenzentwicklung im Schuljahr nach PISA 2012: Effekte von Klassenwiederholungen (Ehmke et al. 2017b) die Auswirkungen von Klassenwiederholungen auf die Entwicklung mathematischer Kompetenz sowie mathematikbezogener Motivationen und Einstellungen.

An der Berichtlegung des Projektes PISA-Plus 2012-2013, welche nun im Rahmen eines Sonderhefts der Zeitschrift für Erziehungswissenschaft erfolgt, beteiligten sich alle drei ZIB-Standorte und weitere Verbundpartner sowie mehrere externe Expertinnen und Experten. Die in diesem Sonderheft vorliegenden Beiträge sind in enger Kooperation standortübergreifender Autorengruppen des ZIB entstanden. Zudem stellt PISA Plus ein Beispiel für die gelungene Zusammenarbeit und Kooperation mit dem IQB und weiteren externen Partnern dar. Im Einzelnen beteiligten sich am Projekt PISA Plus 2012-2013 die folgenden Kooperationspartner.

- School of Education - Technische Universität München (ZIB-Standort München)

- Deutsches Institut für Internationale Pädagogische Forschung - DIPF (ZIB-Standort Frankfurt) 
- Leibniz-Institut für die Pädagogik der Naturwissenschaften und Mathematik IPN

(ZIB-Standort Kiel)

- Institut zur Qualitätsentwicklung im Bildungswesen - IQB

- Leuphana Universität Lüneburg, Lehrstuhl für Erziehungswissenschaft, insbesondere Empirische Bildungsforschung

(Prof. Dr. Timo Ehmke)

- IEA Hamburg (Stichprobenziehung und Gewichtung)

Allen Kooperationspartnern sowie Autorinnen und Autoren im Projekt PISA Plus sei an dieser Stelle sehr herzlich für ihr ausdauerndes Engagement gedankt.

Den Leserinnen und Lesern wünschen wir eine interessante Lektüre der einzelnen Beiträge und hoffen, dass das vorliegende Sonderheft die eine oder andere Anregung mitgibt.

München, im Mai 2017

Jörg-Henrik Heine

Projektkoordination PISA Plus 2012-2013

\section{Literatur}

Diekmann, A. (2014). Empirische Sozialforschung: Grundlagen (9. Aufl.). Reinbek: Rowohlt.

Ehmke, T., Köller, O., \& Stanat, P. (2017a). Äquivalenz der Erfassung mathematischer Kompetenzen in PISA 2012 und im IQB-Ländervergleich 2012. In K. Reiss, E. Klieme, O. Köller \& P. Stanat (Hrsg.), PISA Plus 2012-2013 Zeitschrift für Erziehungswissenschaften - Sonderheft 33. Wiesbaden: Springer VS.

Ehmke, T., Sälzer, C., Pietsch, M., Drechsel, B., \& Müller, K. (2017b). Kompetenzentwicklung im Schuljahr nach PISA 2012: Effekte von Klassenwiederholungen. In K. Reiss, E. Klieme, O. Köller \& P. Stanat (Hrsg.), PISA Plus 2012-2013 Zeitschrift für Erziehungswissenschaften - Sonderheft 33. Wiesbaden: Springer VS.

Hahnel, C., Goldhammer, F., Kröhne, U., Schiepe-Tiska, A., Lüdtke, O., \& Nagy, G. (2017). Der Einfluss kognitiver Basisfertigkeiten auf die Änderung der in PISA gemessenen Lesekompetenz. In K. Reiss, E. Klieme, O. Köller \& P. Stanat (Hrsg.), PISA Plus 2012-2013 Zeitschrift für Erziehungswissenschaften - Sonderheft 33. Wiesbaden: Springer VS.

Heine, J.-H., Mang, J., Borchert, L., Gomolka, J., Kröhme, U., Goldhammer, F., \& Sälzer, C. (2016). Kompetenzmessung in PISA 2015. In K. Reiss, C. Sälzer, A. Schiepe-Tiska, E. Klieme \& O. Köller (Hrsg.), PISA 2015 Eine Studie zwischen Kontinuität und Innovation (S. 383-430). Münster: Waxmann.

Heine, J.-H., Nagy, G., Meinck, S., Zühlke, O., \& Mang, J. (2017). Empirische Grundlage, Stichprobenausfall und Adjustierung im PISA-Längsschnitt 2012-2013. In K. Reiss, E. Klieme, O. Köller \& P. Stanat (Hrsg.), PISA Plus 2012-2013 Zeitschrift für Erziehungswissenschaften - Sonderheft 33. Wiesbaden: Springer VS.

Kiemer, K., Haag, N., Mülller, K., \& Ehmke, T. (2017). Einfluss sozialer und zuwanderungsbezogener Disparitäten, sowie der Klassenkomposition auf die Veränderung der mathematischen Kompetenz von der neunten zur zehnten Klassenstufe. In K. Reiss, E. Klieme, O. Köller \& P. Stanat (Hrsg.), PISA Plus 2012-2013 Zeitschrift für Erziehungswissenschaften - Sonderheft 33. Wiesbaden: Springer VS. Kromrey, H. (2002). Empirische Sozialforschung (10. Aufl.). Opladen: Leske + Budrich.

Kuger, S., Klieme, E., Lüdtke, O., Schiepe-Tiska, A., \& Reiss, K. (2017). Mathematikunterricht und Schülerleistung in der Sekundarstufe: Zur Validität von Schülerbefragungen in Schulleistungsstudien. In K. Reiss, E. Klieme, O. Köller \& P. Stanat (Hrsg.), PISA Plus 2012-2013 Zeitschrift für Erziehungswissenschaften - Sonderheft 33. Wiesbaden: Springer VS. 
Lehner, M.C., Heine, J.-H., Sälzer, C., Reiss, K., Haag, N., \& Heinze, A. (2017). Veränderungen der mathematischen Kompetenz von der 9. zur 10. Klassenstufe. In K. Reiss, E. Klieme, O. Köller \& P. Stanat (Hrsg.), PISA Plus 2012-2013 Zeitschrift für Erziehungswissenschaften - Sonderheft 33. Wiesbaden: Springer VS.

Nagy, G., Haag, N., Lüdtke, O., \& Köller, O. (2017a). Längsschnittskalierung der Tests zur Überprüfung des Erreichens der Bildungsstandards der Sekundarstufe I im PISA-Längsschnitt 2012/2013. In K. Reiss, E. Klieme, O. Köller \& P. Stanat (Hrsg.), PISA Plus 2012-2013 Zeitschrift für Erziehungswissenschaften - Sonderheft 33. Wiesbaden: Springer VS.

Nagy, G., Lüdtke, O., Heine, J.-H., \& Köller, O. (2017b). IRT-Skalierung der Tests im PISA-Längsschnitt 2012/2013: Auswirkungen von Testkontexteffekten auf die Zuwachsschätzung. In K. Reiss, E. Klieme, O. Köller \& P. Stanat (Hrsg.), PISA Plus 2012-2013 Zeitschrift für Erziehungswissenschaften Sonderheft 33. Wiesbaden: Springer VS.

Nagy, G., Retelsdorf, J., Goldhammer, F., Schiepe-Tiska, A., \& Lüdtke, O. (2017c). Veränderungen der Lesekompetenz von der 9. zur 10. Klasse: Differenzielle Entwicklungen in Abhängigkeit der Schulform, des Geschlechts und des soziodemografischen Hintergrunds? In K. Reiss, E. Klieme, O. Köller \& P. Stanat (Hrsg.), PISA Plus 2012-2013 Zeitschrift für Erziehungswissenschaften - Sonderheft 33. Wiesbaden: Springer VS.

Pant, H.A., Stanat, P., Schroeders, U., Roppelt, A., Siegle, T., \& Pohlmann, C. (Hrsg.). (2013). IQBLändervergleich 2012: mathematische und naturwissenschaftliche Kompetenzen am Ende der Sekundarstufe I. Münster: Waxmann.

PISA-Konsortium Deutschland (Hrsg.). (2006). Pisa 2003 Untersuchungen zur Kompetenzentwicklung im Verlauf eines Schuljahrs. Münster: Waxmann.

Prenzel, M., Sälzer, C., Klieme, E., \& Köller, O. (Hrsg.). (2013). PISA 2012 - Fortschritte und Herausforderungen in Deutschland. Münster: Waxmann.

Schiepe-Tiska, A., Rönnebeck, S., Heitmann, P., Schöps, K., Prenzel, M., \& Nagy, G. (2017). Die Veränderung der naturwissenschaftlichen Kompetenz von der 9. zur 10. Klasse bei PISA und den Bildungsstandards unter Berücksichtigung geschlechts- und schulartspezifischer Unterschiede sowie der Zusammensetzung der Schülerschaft. In K. Reiss, E. Klieme, O. Köller \& P. Stanat (Hrsg.), PISA Plus 2012-2013 Zeitschrift für Erziehungswissenschaften - Sonderheft 33. Wiesbaden: Springer VS. 